\title{
FÍSICA DEL LÁSER
}

\author{
J. M. Banús Gassol.
}

ICUN. Instituto Catalán de Urología y Nefrología. Barcelona. España.

\begin{abstract}
Resumen.- El encargo de este pequeño artículo me sumió en la duda. En primer lugar debo confesar que no encuentro excusa para saltarse esta parte si alguien quiere profundizar mínimamente en el conocimiento de los efectos biológicos de esta fuente de energía. En segundo lugar, cuando hablamos de los resultados, usamos términos acuñados y definidos por la física. A menudo polemizamos sobre resultados y lo que en realidad ocurre es que no nos ponemos de acuerdo porque nos referimos a términos distintos para explicar una misma observación; en definitiva, no logramos entendernos porque no conocemos los términos adecuados; por ejemplo, hipoxemia como déficit de oxígeno, que tanto es verdad en un paciente anémico como en una baja saturación de oxígeno. En consecuencia, me parece imprescindible un buen repaso a estos conceptos. La tercera razón es el confusionismo que existe en nuestro medio, a veces pienso que interesado, sobre propie-
\end{abstract}

dades y efectos de diferentes tipos de láser. Solo un mínimo conocimiento de la física nos ayudará a sentar las bases científicas para entendernos.

Los problemas, sin embargo, se acumulan, dado que el universo al que va dirigido este pequeño artículo está formado por urólogos. ¿¿Qué formación en física debemos suponerles? ¿Superficial? ¿Media? ¿ Será un colectivo con un conocimiento uniforme, sea éste el que sea? La implicación es clara. La profundidad del artículo dependerá de las respuestas que demos a las preguntas anteriores.

No obstante, el ánimo de los autores consiste en dar una base suficiente como para entender qué es y cómo actúa el láser. Por ello, la respuesta que me he dado es que el lector debe entender el artículo, y tener una base suficiente, como mínimo para leer críticamente los artículos sobre láser que se publican en las revistas urológicas.

Palabras clave: Física. Láser.

Summary.- The commission of this article plunged me into doubt. First I should confess that I don't find excuse to escape this part if somebody wants to minimally deepen in the knowledge of the biological effects of this energy source. Secondly, when we talk about results, we use terms made and defined by Physics. Often we have polemics about results, and what really happens is that we don't reach agreements because we refer to different terms to explain the same observation; in conclusion we cannot understand each other because we do not know the adequate terms; for example, hypoxemia as oxygen deficit, which is true in an anemic patient as well as in a low oxygen saturation rate. In consequence, a good review of these concepts seems necessary to me. The third reason is the confusion that exists in our environment, I think sometimes of interest, about properties and effects of different types of laser. Only a minimal knowledge of physics will help us to state the scientific basis for understanding. 
The problems, nevertheless, accumulate due to the fact that the universe to which this article is directed is formed by urologists. What Physics education should we suppose they have? Superficial? Medium? Is it a collective with a uniform knowledge, being it whatever it is? The implication is clear. The article depth will depend on the answers to these questions.

Nevertheless, the aim of the authors is to give a base enough to know what the laser is and how it acts. For that, the answer I gave to my questions is that the reader should understand the article and have enough base for, at least, reading critically the articles about laser published in urological journals.

\section{Keywords: Physics. Laser.}

\section{DEFINICIÓN}

Láser es un acrónimo (palabra formada por las letras o segmentos iniciales -o finales- de las palabras que componen una frase) del inglés Light Amplification by Stimulated Emission of Radiation.

\section{HISTORIA}

1917: Einstein propuso el concepto de emisión estimulada de radiación.

1960: Maimen, T.H. usó esta teoría para fabricar la primera luz visible de láser usando un cristal sintético de rubidio con sus extremos envueltos en plata y colocándolo dentro de un tubo de flash para producir energía.

1966: Parsons usa este mismo láser para trabajar experimentalmente en vejigas caninas.

1968: Mulvany intenta fragmentar cálculos con este mismo láser.

\section{FísICA}

cos sobre el láser.

Debemos a Albert Einstein los dos principios bási-

El primero establece que la luz viaja en el espacio en forma de paquetes conocidos con el nombre de fotones.

El segundo explica que en el átomo formado por protones y neutrones en el núcleo, que está rodeado de electrones, estos se disponen en distintos niveles (lo que Max Plank definió como órbitas). Cuando se introduce energía en este sistema, estos electrones puede "migrar" a una órbita (o a un estado) superior. En estado excitado, estos electrones pueden pasar espontáneamente a su estado basal. Cuando esto ocurre, se libera energía electromagnética del sistema que, de acuerdo a la primera definición, viajará en forma de fotones.
Si encerramos estos átomos entre espejos, los fotones rebotarán en los espejos y chocarán con otros átomos, los electrones de los cuales pasarán de su estado basal a estar excitados, de forma que la mayoría de los átomos estarán en estado de excitación. Esto se conoce como inversión poblacional (o population inversion en inglés).

A su vez, estos electrones excitados tenderán a volver a su estado natural, liberando más fotones. Precisamente este es el principio que explica la formación del haz de energía del láser.

De estos principios de física se desprenden tres grandes conclusiones, que podríamos llamar las tres $C$ del láser:

1. Coherencia. Suponiendo una fuente para el láser con átomos iguales, iguales serán las cantidades de energía obtenidas con el "salto" del electrón hacia su estado original, de ahí que la frecuencia del haz sea coherente.

2. Cromatismo. El haz es monocromático. Esto es, si hacemos pasar el haz a través de un prisma, el haz entrante en él y el saliente serán idénticos.

3. Colimado. El haz no se dispersa a medida que se aleja de la fuente, como ocurre con el resto de haces luminosos.

\section{ANATOMÍA DEL LÁSER}

Para la generación del haz de energía, debemos disponer de un átomo que no ponga problemas para dejar excitar los electrones de sus últimas órbitas. Este material, conocido como el medio del láser es el que da el nombre a éste (p. ej. Rubidio, CO2, holmio,...). Este material se coloca en una caja de resonancia óptica, que está construida mediante dos espejos. Uno de ellos es completo, es decir, refleja el $100 \%$ de la luz que le llega, y el otro es parcial, reflejando solo parte de los fotones, porque por el agujero que se ha construido saldrá hacia el exterior el haz de luz.

Como vemos en la Figura 1, existe una fuente de energía que funciona como estimulador. Los electrones

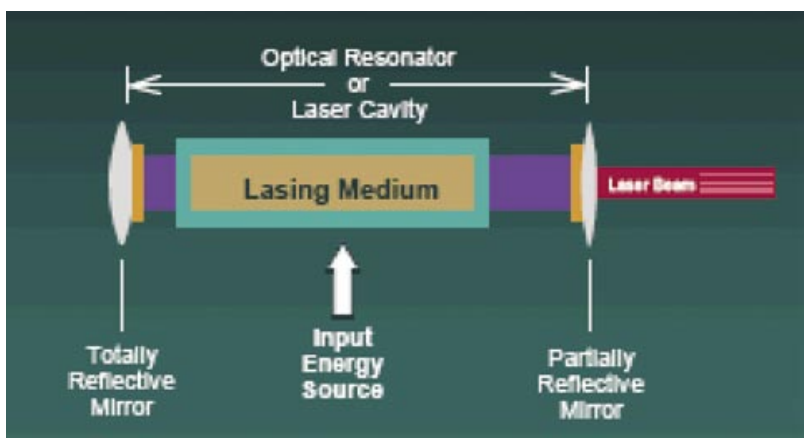

FIGURA 1. Esquema de la generación de la energía láser. 
del átomo, en situación normal, tienden a estar en el nivel más bajo posible de energía. Sin embargo, si aportamos al sistema energía electromagnética, ya sea mediante descargas eléctricas, o luz (fotones), esta será absorbida por la fuente del láser y excitará un número determinado de electrones. Cuando un electrón excitado vuelva a su estado de reposo, emitirá un nuevo fotón. Este fotón, si choca con otro átomo, excitará otros electrones, que a su vez emitirán nuevos fotones, con la particularidad que todos ellos viajarán en la misma dirección. Cuando choquen con el espejo, invertirán su sentido hacia el espejo de salida, el que presenta una abertura, por el que saldrá hacia el exterior un haz de fotones, todos ellos viajando en la misma dirección (colimados), se encontrarán en la misma fase (coherentes) y tendrán la misma longitud de onda (monocromáticos). Este será el haz del láser.

¿Qué ocurre con los fotones que se han vuelto a reflejar en el espejo de salida? Estos fotones vuelven hacia el interior de la fuente del láser, para, a su vez, chocar contra otros átomos de la fuente, estimularlos, y generar nuevos fotones, de manera que la población de átomos estimulados será cada vez mayor, y por lo tanto la generación de fotones al pasar a su estado de reposo será también mayor. Este fenómeno, conocido como inversión poblacional, o population inversion es fundamental para la generación del láser.

A menudo los láseres tienen un nombre compuesto de dos sustancias. Cuando ello ocurre, deberíamos pensar que deberían producir dos tipos de fotones. En realidad, así es, aunque el haz de láser sea de una sola longitud de onda. Lo que ocurre es que uno de los componentes se usa como fuente de fotones para estimular al otro componente, que es el que, de hecho, producirá el haz del láser.

Diferentes medios (átomos) emitirán protones a diferentes longitudes de onda. Las propiedades que tendrá el láser serán fundamentalmente debidas a dicha longitud de onda.

\section{PROPIEDADES QUE DEFINEN EL LÁSER}

Energía: Se mide en joules y describe la cantidad de trabajo que realiza.

Potencia: Describe la cantidad de trabajo por unidad de tiempo de puede realizar, y se mide en watts o en joules/ segundo.

Fluencia: También conocida como densidad de potencia, expresa la cantidad de trabajo realizado en una superficie determinada (joules $/ \mathrm{cm}^{2}$ ).

Irradiancia: Describe la intensidad de la fuente de láser y se mide en watts por $\mathrm{cm}^{2}$, y es inversamente proporcional al cuadrado del radio de la fibra. Colocando una lente que concentre o desenfoque el haz del rayo, podemos cambiar la cantidad de luz que recibe un mismo territorio de tejido, incluso manteniendo la fibra a una misma distancia del tejido diana.
Por otro lado, el haz de láser puede salir de la fuente de láser de tres formas posibles:

Continuo: Asumimos que, aparte de pequeños problemas técnicos (por ejemplo sobrecalentamiento de la cavidad donde se forma el láser) la cantidad de energía que obtenemos de la fuente de láser es constante a lo largo del tiempo.

Pulsada: En este caso la energía obtenida se subdivide en pulsos, lo que produce por ejemplo menor cantidad de calor que se dispersa en el tejido, y por tanto el efecto sobre el tejido es más "puro". Idealmente la cantidad de energía emitida en cada pulso no debe ser mayor que si ésta fuera emitida de forma continua. La fuente del láser actúa estimulando los átomos entre pulsos, por lo que la energía obtenida en el pico es mayor que si la misma fuente emitiera de forma continua. Existe una variante en este apartado, denominada superpulso, en el que los pulsos son tan cortos y tan frecuentes (entre 300 y 1000 por segundo), que la emisión de energía actúa en realidad de forma continua.

Modo Q-switched: Esta técnica produce picos de energía de gran intensidad (del orden de decenas de millones de watts) de muy escasa duración (unos pocos nanosegundos). Esto permite que exista muy poca dispersión colateral de calor y un efecto más directo y preciso).

Finalmente, el uso obligado de fibras en nuestro medio que permitan transportar la luz del láser hasta el lugar donde queremos obtener un determinado efecto, introduce otro efecto físico que conviene conocer.

A menudo la fibra permite una divergencia de la luz de unos 10 - 15을 desde la salida de la fuente hasta la punta de la fibra. Esta defocalización se incrementa si retiramos la punta de la fibra del tejido diana sobre el que deseamos actuar. Apartando la punta de la fibra del tejido hasta una pulgada de distancia, podemos obtener efectos muy diversos, desde cortar el tejido (máxima concentración de luz: la punta toca el tejido), hasta vaporizar la superficie de éste (ligeramente desenfocado), hasta obtener una coagulación proteica (y por tanto un mayor poder de coagulación), cuando alejamos algo más la fibra.

En el capítulo de interacción del láser con el tejido se suele centrar el tema en la longitud de onda de cada láser para predecir su efecto sobre el tejido, y sobre todo la penetración del láser. Efectivamente la absorción de la energía por el tejido depende mayoritariamente de la longitud de onda del láser, pero no debemos olvidar que la energía que aplicamos, aparte del efecto "mecánico" que deseamos, se dispersará en forma de calor, y que uno de los efectos colaterales que debemos esperar de una "quemadura" en el tejido, es la necrosis del mismo, y su posterior absorción, mediante un proceso inflamatorio. La rapidez en la recuperación del paciente, y en su caso (por ejemplo en la próstata) la desaparición de la sintomatología inflamatoria dependerá del cariño con que tratemos el tejido, y del menor efecto inflamatorio que seamos capaces de generar. 
De forma general, por tanto, podríamos establecer que a mayor energía administrada al tejido, mayor será este efecto, y que éste tenderá a reducirse usando menores cantidades de energía, y/o energía suministrada en forma de pulsos, dado que asi disminuye la energía colateral dispersada en forma de calor.

\section{CONCLUSIONES}

El uso de un equipamiento médico se supone que obliga (aunque sea moralmente) a un mínimo conocimiento de sus principios. De ahí el interés para que pueda difundirse en nuestro medio unos conceptos de física sobre el láser. En el prólogo de su primer libro de divulgación Stephen Hawkings comentaba que su editor le había pronosticado que por cada fórmula que pusiera en el texto, perdería por lo menos un potencial lector. Él puso sólo una: la conocida de Einstein de la energía y la masa. Yo no me he atrevido a poner ninguna, para que no sea esta la causa de que algún lector abandone la lectura de este artículo. Seguro que encontrará otras muchas excusas.

Ya me perdonará que no se las detalle; así, al menos tendrá que leerlo un poco para encontrarlas él mismo.

\section{BIBLIOGRAFÍA y LECTURAS RECOMENDADAS ("lectura de interés $y^{* *}$ lectura fundamental)}

**1. http://www.wikipedia.org

**2. http://www.howstuffworks.com

3. Wodsworth, W. Easy Laser Physics. Mht In: http://www.physics.ox.ac.uk/al/research/groups/laser/ lasers.html

4. Weschler, M.: How Laser Work, in: How Stuff Works. 